\title{
Allemagne. Une prise de conscience tardive
}

Étude de cas

\section{Hans-Konrad Koch}

\section{CpenEdition}

\section{Journals}

Édition électronique

URL : https://journals.openedition.org/ries/1233

DOI : $10.4000 /$ ries. 1233

ISSN : 2261-4265

Éditeur

France Education international

Édition imprimée

Date de publication : 1 décembre 2005

Pagination : 27-29

ISSN : 1254-4590

Référence électronique

Hans-Konrad Koch, "Allemagne. Une prise de conscience tardive ", Revue internationale d'éducation de Sèvres [En ligne], 40 | décembre 2005, mis en ligne le 10 avril 2012, consulté le 07 juillet 2021. URL : http://journals.openedition.org/ries/1233; DOI : https://doi.org/10.4000/ries.1233

Ce document a été généré automatiquement le 7 juillet 2021.

(c) Tous droits réservés 


\section{Allemagne. Une prise de conscience tardive}

Étude de cas

Hans-Konrad Koch

1 En matière d'évaluation, l'Allemagne a une tradition beaucoup plus limitée que celle de la France et d'autres pays comparables. Mais ces dernières années, la question de l'évaluation des établissements scolaires a vu, en Allemagne aussi, son importance grandir considérablement, pour deux raisons notamment. On voit maintenant, plus tard que dans d'autres pays, se multiplier les débats sur une plus grande autonomie de l'école. L'accès des établissements scolaires à l'autonomie va de pair avec l'utilisation de l'outil qu'est l'évaluation pour garantir et améliorer la qualité des cours. D'autre part, les résultats des enquêtes internationales sur les résultats scolaires comme TIMSS, PISA et IGLU ont, depuis la fin des années quatre-vingt-dix, toutes remis en question la qualité du système éducatif allemand dans son ensemble. En conséquence, on accorde une grande importance à, notamment, la recherche empirique sur l'enseignement, l'observation permanente sur la base d'indicateurs du système scolaire ainsi que le développement d'une culture de l'évaluation au niveau des établissements.

\section{PISA, le déclic pour une nouvelle réforme}

2 Fin 2001, les mauvais résultats obtenus par les élèves allemands à l'enquête PISA ont causé un choc. Experts et hommes politiques savaient depuis longtemps que l'enseignement était une question d'avenir et qu'il fallait réformer le système éducatif. Mais il a fallu attendre ces résultats pour que l'opinion publique prenne conscience des faiblesses du système éducatif et de la nécessité d'une nouvelle réforme de l'enseignement. Cette prise de conscience a été le préalable à une action politique.

On peut résumer ainsi les résultats allemands lors de l'étude PISA 2003 :

- dans les trois domaines de compétences qui ont fait l'objet de l'évaluation, le score global des élèves allemands se trouve au minimum dans la moyenne des trente pays de l'OCDE ; lors de PISA 2000, leurs résultats étaient inférieurs à la moyenne ; 
- de façon inchangée, le groupe à risque de ceux qui atteignent au maximum le niveau de compétences le plus bas est de $23 \%$;

- l'Allemagne est, parmi tous les pays industriels comparables, le pays avec la plus étroite corrélation entre l'origine sociale et les résultats scolaires ;

- comparée aux autres pays de l'OCDE, la dispersion des résultats entre les établissements est très grande.

\section{Objectifs de la réforme}

La conséquence de ces mauvais résultats est qu'il existe aujourd'hui, en Allemagne, un consensus sur la nécessité d'une nouvelle et profonde réforme de l'enseignement. L'objectif est d'améliorer durablement la qualité du système éducatif allemand et de réduire enfin l'écart dramatique entre l'origine sociale et les résultats scolaires. La réalisation de ces objectifs suppose un changement d'état d'esprit radical. Il faut passer d'un système éducatif par tradition très sélectif à un système qui encourage les élèves tout en exigeant des résultats, qui accorde la priorité au développement de chaque enfant et de chaque adolescent en s'appuyant sur ses points forts.

Entre-temps, le gouvernement fédéral et les Länder ont pris toute une série de mesures, en particulier dans les domaines suivants :

- la promotion précoce : outre l'augmentation du nombre de crèches selon les besoins, la mission éducative du jardin d'enfants et la qualification des personnels ont la priorité ;

- la multiplication des écoles à temps plein : d'ici 2007, le gouvernement fédéral va mettre à la disposition des Länder des crédits d'investissements d'un montant de quatre milliards d'euros pour la multiplication des écoles à temps plein en fonction des besoins; ces nouvelles écoles à temps plein doivent faire émerger une nouvelle culture d'apprentissage et d'enseignement centrée sur le développement individuel de chaque enfant;

- l'amélioration des cours en termes de contenus et de méthodes: des modèles de programmes communs au gouvernement fédéral et aux Länder ont pour objectif de renforcer les compétences en mathématiques et en sciences naturelles, d'améliorer la compréhension de l'écrit, de promouvoir systématiquement les enfants de familles d'immigrants ainsi que de renforcer le développement précoce et individuel ;

- l'introduction de standards éducatifs nationaux : en 2004-2005, les Länder ont commencé à introduire des standards éducatifs nationaux dans les disciplines suivantes: allemand, mathématiques, première langue étrangère et sciences naturelles ;

- la professionnalisation des personnels: le succès de la réforme de l'enseignement en dépend; la formation initiale et la formation continue doivent préparer à de nouvelles missions telles que le développement individuel, la coopération avec des partenaires dans le domaine de l'éducation informelle, la mise en place de l'autonomie des établissements scolaires et l'évaluation;

- un rapport national sur le système éducatif : à l'issue de longues années de négociations, le gouvernement fédéral et les Länder se sont mis d'accord sur un rapport commun, régulier et indépendant qui portera sur l'ensemble du système éducatif, y compris l'éducation non formelle et informelle.

\section{Vers la généralisation de l'évaluation}

6 La mise en œuvre et le succès de cette nouvelle réforme de l'enseignement sont inimaginables sans l'évaluation. L'évaluation est un aspect essentiel d'une nouvelle 
gestion du système qui tient compte, de plus en plus, des résultats (output) dans les processus éducatifs.

7 L'évaluation par des enquêtes internationales comme PISA et IGLU est entre-temps devenue une base incontournable pour le processus de réforme de l'enseignement en Allemagne.

8 Début 2006, un consortium indépendant de scientifiques va, pour la première fois, présenter un rapport sur l'ensemble du système éducatif allemand qui s'appuiera sur des indicateurs. Ce rapport montrera aux politiques, à l'administration et à l'opinion publique si les mesures prises commencent à avoir de l'effet et dans quels domaines il faut les compléter. À long terme, il est prévu que ce rapport soit soutenu par un dispositif national d'évaluation qui pourrait débuter en 2009 sous la forme d'études séquentielles de cohortes et est censé être compatible avec les enquêtes comparatives internationales.

9 En 2006 sera graduellement mise en place l'évaluation des nouveaux standards éducatifs nationaux. Pour assurer une mise en œuvre de la normalisation et de l'évaluation à un niveau commun à tous les Länder, ces derniers ont créé l'Institut d'assurance de la qualité dans le système éducatif. Parallèlement, quatorze instituts seront créés dans les Länder.

10 À l'heure actuelle, de grands projets de recherche sont en cours d'élaboration afin d'améliorer les bases de l'évaluation des compétences. L'objectif est de savoir comment définir les compétences et comment les évaluer en s'appuyant sur l'ordinateur et l'Internet.

11 Pour le moment, le terme "évaluation " suscite encore de profondes craintes, en particulier parmi les enseignants. Beaucoup d'entre eux, mais aussi d'élèves et de parents, ne comprennent pas encore la grande chance que l'évaluation interne et externe représente pour l'émergence d'une nouvelle culture de l'apprentissage et de l'enseignement. Mais toute une série d'exemples montrent que, en Allemagne aussi, le développement d'une culture de l'évaluation est lancé.

INDEX

Index géographique : Allemagne

\section{AUTEUR}

HANS-KONRAD KOCH

Ministère fédéral de l'Education et de la Recherche, directeur de la réforme du système éducatif. 